MAJOR ARTICLE

\title{
A Cohort Study of the Effectiveness of Influenza Vaccine in Older People, Performed Using the United Kingdom General Practice Research Database
}

\author{
Punam Mangtani, ${ }^{1}$ Phillippa Cumberland, ${ }^{1, a}$ Cathy R. Hodgson, ${ }^{2}$ Jennifer A. Roberts, ${ }^{1}$ Felicity T. Cutts, ${ }^{3}$ \\ and Andrew J. Hall' \\ 'London School of Hygiene and Tropical Medicine and ${ }^{2}$ Office for National Statistics, London, United Kingdom; ${ }^{3}$ Medical Research Council \\ Laboratories, Banjul, Gambia
}

Background. The effectiveness of influenza vaccination against hospitalization and death can only ethically be assessed in observational studies. A concern is that individuals who are vaccinated are healthier than individuals who are not vaccinated, potentially biasing estimates of effectiveness upward.

Methods. We conducted a historical cohort study of individuals $>64$ years of age, for whom there were data available in the General Practice Research Database for 1989 to 1999 in England and Wales. Rates of admissions for acute respiratory diseases and rates of death due to respiratory disease were compared over 692,819 personyears in vaccine recipients and 1,534,280 person-years in vaccine nonrecipients.

Results. The pooled effectiveness of vaccine against hospitalizations for acute respiratory disease was $21 \%(95 \%$ confidence interval [CI], 17\%-26\%). The rate reduction attributable to vaccination was 4.15 hospitalizations/100,000 person-weeks in the influenza season. Among vaccine recipients, no important reduction in the number of admissions to the hospital was seen outside influenza seasons. The pooled effectiveness of vaccine against deaths due to respiratory disease was $12 \%$ (95\% CI, 8\%-16\%). A greater proportionate reduction was seen among people without medical disorders, but absolute rate reduction was higher in individuals with medical disorders, compared with individuals without such disorders (6.14 deaths due to respiratory disease/100,000 person-weeks vs. 3.12 deaths due to respiratory disease/100,000 person-weeks). Clear protection against death due to all causes was not seen.

Conclusions. Influenza vaccination reduces the number of hospitalizations and deaths due to respiratory disease, after correction for confounding in individuals $>64$ years of age who had a high risk or a low risk for influenza. For elderly people, untargeted influenza vaccination is of confirmed benefit against serious outcomes.

Influenza can have a dramatic effect on mortality [1]. However, the role of influenza vaccination in preventing death is poorly quantified, because many deaths are due to secondary pneumonia or acute exacerbations of chronic medical conditions, such as chronic lung or heart disease [2], and because the role of influenza in death is not routinely documented in mortality statistics $[3,4]$. Only observational studies of protection by in-

Received 19 August 2003; accepted 23 December 2003; electronically published 26 May 2004.

Financial support: Department of Health project grant; Wellcome Trust (grant GR0051637 to P.M.).

a Present affiliation: Institute of Child Health, London, United Kingdom.

Reprints or correspondence: Dr. Punam Mangtani, London School of Hygiene and Tropical Medicine, Keppel St., London WC1E 7HT, UK (punam.mangtani@Ishtm .ac.uk).

The Journal of Infectious Diseases 2004; 190:1-10

(C) 2004 by the Infectious Diseases Society of America. All rights reserved. 0022-1899/2004/19001-0001\$15.00 fluenza vaccination against more serious outcomes exist. One of these studies, a recent historical cohort study performed in the United States during the winter of 1998-1999, suggested that influenza vaccination had a protective effect of $48 \%$ (95\% confidence interval [CI], $43 \%-53 \%$ ) against death due to all causes [5]. Another study, which was performed in the United Kingdom during the influenza epidemic of 1989-1990 and which was based on much smaller numbers of patients, noted that the effectiveness of influenza vaccine was $74.7 \%$ (95\% CI, 21.2\%-91.9\%) [6]. One of the main issues associated with such observational studies is that individuals who are vaccinated tend to be healthier than individuals who are not vaccinated; this may potentially lead to overestimation of the effectiveness of the vaccine [7]. Randomized trial data indicate that, for elderly individuals, influenza vaccination is only $58 \%$ effective in preventing virologically confirmed clinical influenza 
[8]; therefore, large effects of vaccination on mortality appear to be unlikely.

Yearly vaccination is required because of viral antigen drift and unpredictable, occasional antigenic shifts that make existing antibody levels in the population no longer protective. The UK program of targeted influenza vaccination for people with underlying medical disorders was expanded in 1998 to include all people $\geqslant 75$ years of age, and, then, in 2000 , the program was expanded again to include all people $>64$ years of age. We conducted a historical cohort study of the effectiveness of annual vaccination of persons $>64$ years of age over a number of epidemic and nonepidemic years.

Influenza $\mathrm{A}\left(\mathrm{H}_{3} \mathrm{~N}_{2}\right)$ was the predominant subtype noted during 7 of the 10 years from 1989 to 1999. The 3 influenza B seasons occurred in 1990-1991, 1992-1993, and 1994-1995. Only 2 of the influenza B seasons (1992-1993 and 1994-1995) were considered to be seasons during which influenza was mild, as was 1 of the influenza A seasons (1997-1998). In all years, the Public Health Laboratory Service (part of the United Kingdom's Health Protection Agency since 1 April 2003) recorded a very good match between the influenza strains that were circulating and the vaccine strains that were used, except for the 1997-1998 season, for which the $\mathrm{H}_{3} \mathrm{~N}_{2}$ strain was antigenically different from that used in the vaccine and was reported with increased frequency as the season progressed $[9,10]$.

\section{METHODS}

General Practice Research Database (GPRD) data set. In the United Kingdom, general practitioners (GPs) provide, for a defined list of patients, all ambulatory care as well as referrals for hospital-based care provided by specialists. The UK GPRD is an ongoing computerized data set of the routine records of GPs from several hundred selected practices. All data on prescriptions and vaccinations, together with any data on severe illness requiring referral to the hospital and data on deaths, have been obtained since 1989-1990 or since the time when individual practices joined the scheme. Routine recording of hospitalizations began in April 1990. Records of clinical activities are complete and have good validity [11] for prescriptions [12], hospitalizations [12, 13], and morbidities not necessarily resulting in a prescription [14]. Mortality rates from the GPRD are similar to national rates (S. Jick, unpublished data). In GPRD records, information on any medical condition noted on the day of death accords well with information on death certificates. Of information on 170 deaths noted in the GPRD, only information on 11 of these deaths was not concordant with the information on the immediate or underlying cause of death noted on the death certificate (S. Jick, unpublished observations). For technical reasons, general practices that used ViSion software (In Practice Systems), which was introduced in 1995, were not included in the GPRD at the time that the present study was conducted.

The general practices included in the study were restricted to England and Wales, because hospitalization patterns in these countries vary from those in Scotland. In 1993, these practices represented 3.8\% of the population of England and Wales [15]. The subjects are generally representative of the population in terms of age and sex $[15,16]$. Data on individual socioeconomic status were not available.

The present study linked influenza vaccination to subsequent hospitalization and death, for individual patients registered in these practices over 10 years. Patients $>64$ years of age were identified and included in the study if they were registered on the first day of the week that included 1 September each year. These patients contributed person-time to the study until either death or "transfer out" of the practice.

End points. Diagnoses of acute respiratory illnesses (i.e., influenza, pneumonia, and acute bronchitis/bronchiolitis [International Classification of Diseases-Ninth Revision, or ICD-9, codes 487, 480-486, and 466, respectively]) that were considered to be the reason for hospitalization were judged to be the most appropriate marker of admissions to the hospital for pneumonia and influenza, because it is difficult to distinguish clinically between these 3 syndromes. Seventy percent of all deaths that were defined, according to ICD-9 criteria, as being respiratory related (i.e., "respiratory chapter deaths") were reported as acute respiratory illnesses. Because a substantial minority of GPs could have noted only an underlying respiratory cause for influenza-associated deaths, all respiratory-related deaths were examined.

Exposures. Person-weeks that followed 15 November each year were designated as "vaccinated" or "not vaccinated," according to whether subjects had been vaccinated against influenza at the start of each week. In all years except 1989-1990 (i.e., $77 \%$ of years assessed), $>85 \%$ of vaccine recipients had been vaccinated by 15 November.

To assess the effect of expansion of influenza vaccination, in 1998 , to all individuals $>64$ years of age, subjects were stratified into 2 groups according to the degree of risk for influenza. The high-risk group had medical disorders that most closely matched Joint Committee on Vaccination and Immunisation (Department of Health, London, United Kingdom) recommendations for targeted influenza vaccinations up to 1998 [17]. These disorders included chronic respiratory disease, including asthma; cardiovascular, renal, and liver disease; immunosuppressive conditions; and metabolic diseases, such as diabetes.

Controlling for possible confounders. To control for the presence and severity of underlying illness in vaccinated individuals, compared with individuals who were not vaccinated, we extracted data on the number of repeat prescription items 
for drugs for the cardiovascular, respiratory, and endocrine systems; the central nervous system; infections; malignant disease; and immunosuppression; as well as for nutrition and blood [18] (also available at http://www.bnf.org) during the preceding 12 months. Rates of hospitalization increased with increasing numbers of prescriptions $(P<.001$, for linear trend), for both high- and low-risk subjects; this finding confirmed that repeat prescriptions would be a good marker of underlying ill health requiring hospital care. Information on smoking was not used in the present study, because there was incomplete and biased recording of such information in the GPRD for patients $>64$ years of age (GPRD, unpublished data).

The effect of influenza vaccine on mortality and hospitalization rates was also stratified by season, to assess for the presence of confounding from factors other than from underlying illness (i.e., for effects resulting from unmeasured differences between vaccinated and unvaccinated individuals). If unmeasured differences existed (e.g., if lower levels of smoking were noted for vaccine recipients), an apparent effect of the influenza vaccine would be seen in either the peri-influenza season or the summer season. Because it is plausible that some confounders might have a greater effect in winter than in summer (e.g., because of an association with other respiratory infections that occur in the winter, such as respiratory syncytial virus), we assigned more importance to the peri-influenza season, rather than the summer season, to assess the effectiveness of influenza vaccination. The "influenza season" in each year was defined using GP consultation rates for influenza-like illness that has been validated against the presence of circulating influenza virus in the same population [19]. In this surveillance system, the onset and the end of the influenza season occurred when consultation rates for influenza-like illness increased to $\geqslant 50$ consultations/ 100,000 person-weeks and decreased to $<50$ consultations/100,000 person-weeks, respectively [19]. The "periinfluenza season" was defined as the weeks during the winter period that were outside the influenza season from 15 November each year to the end of April. The end of April was the latest end point of one of the influenza seasons in the 10-year period. The "summer season" was defined as the period from 1 May to the end of August each year.

Statistical analysis. Poisson regression was used to obtain rate ratios (RRs) for vaccinated individuals and for individuals who were not vaccinated against influenza, by use of data pooled over the course of 10 years. Analyses by year were also performed to check for consistency. Confounding was assessed by analyses of the RR for vaccinated individuals, compared with nonvaccinated individuals, adjusted for each baseline characteristic separately and compared with the unadjusted RR. All variables that were found to have a confounding effect and/or were found to be significant $(P=.1)$ were included in the final model.
It was assumed a priori that influenza vaccination would be protective in the influenza season and not in the peri-influenza season or summer season (i.e., the season acts as an "effect modifier" on influenza vaccination). The presence of other possible effect modifiers was explored using interaction terms. The interpretation of any statistically significant effect modification took into account the large sample size of 1.7 million personyears over the course of 10 years, which could generate statistically significant results that were not necessarily of public health relevance. Important effect modification was therefore decided by (1) a difference in RRs across the strata of the variable set, a priori, with a minimum absolute difference of $10 \%$ in the RRs and (2) statistically significant interaction terms in $\geqslant 7$ of the 10 years in the analyses, by year. This was thought to be appropriate, because, in some years, not much influenza activity was seen. Any variables that met the set criteria were then fitted as interaction terms in the final model. The RRs were estimated separately for each level of the variables that modified the effect of influenza vaccination, adjusted for the remaining confounding variables.

Vaccine effectiveness was calculated as $(1-R R) \times 100$. The 95\% CIs were calculated similarly. If the RRs were lower in the influenza seasons than in the peri-influenza seasons, additional evaluation of the reduction in the rates of hospitalization for respiratory disease attributable to influenza vaccination (i.e., attributable rate reduction) was done. Directly standardized rates, which were standardized for risk category, sex, and repeat prescriptions, were first calculated for the vaccinated cohort, compared with the unvaccinated cohort. The standard population used was the whole cohort. The attributable rate reductions were then calculated as the difference in the directly standardized rates of hospitalization for vaccinated individuals, compared with unvaccinated individuals. The CI for the difference was calculated using the variance for the difference between the standardized rates, based on the sum of the weighted variances of both rates. The data were analyzed using STATA 7 (StataCorp) [20].

\section{RESULTS}

Overall vaccination coverage at the start of each winter season, for all individuals $>64$ years of age, increased from $18 \%$ in $1989-1990$ to $42.6 \%$ in 1998-1999. Each year, and when data were pooled over 10 years, vaccine recipients were more likely than nonrecipients to be older $(P<.001)$, to have underlying medical disorders $(P<.001)$, and to belong to the category of patients with the highest number of repeat prescriptions $(P<$ $.001)$. They also were less likely to have had no repeat prescriptions $(P<.001)$. Approximately $4 \%$ of influenza vaccine recipients and individuals who did not receive influenza vaccine had not been registered for 1 year before 1 September. These individuals were excluded from further analyses. 
Effectiveness of influenza vaccine against hospitalizations. Crude rates of admission to the hospital for acute respiratory disease were higher among vaccinated persons than among unvaccinated persons. After adjustment for risk, age, and repeat prescription status, however, vaccine effectiveness was $21 \%$ (95\% CI, 17\%-26\%) in the influenza season, over the 9 years of varying influenza activity. No important effect of the vaccine against hospitalizations for acute respiratory disease was seen outside the influenza season (table 1). The effectiveness of influenza vaccine was also lower in years in which influenza activity was considered to have been mild. No important effect modification of vaccination by age was found. The effect modification by category of risk was considered to be of borderline importance, because it was seen in 6 of the 9 years of varying influenza activity (data not shown), and, over all years, the difference was small and nonsignificant (table 2 and figure 1). The attributable rate reductions per 100,000 person-weeks were 5.44 (95\% CI, 4.81-6.07) in the influenza season, 1.29 (95\% $\mathrm{CI}, 0.75-1.82)$ in the peri-influenza season, and 0.29 (95\% CI, -0.19 to 0.76 ) in the summer season.

Effectiveness of influenza vaccine against mortality. Crude rates of respiratory-related death were markedly higher among vaccine recipients, compared with nonrecipients, but less so in the influenza season. Adjusting for confounding by risk, age, and prescription category reduced the crude relative risk of death due to respiratory disease in the influenza season from 1.32 to 0.88 , providing a summary vaccine effectiveness of 12\% (95\% CI, 8\%$16 \%)$ over 10 consecutive years. No effect of influenza vaccine was seen in the other seasons (table 3). Little effect of influenza vaccination on death due to respiratory disease was seen in 2 of 3 nonepidemic years (i.e., 1994-1995 and 1997-1998). There was a suggestion of a protective effect against death due to respiratory disease in the third year during which influenza activity was considered to be mild (1992-1993). In 1 year (1995-1996), the effectiveness of influenza vaccine against death due to respiratory disease was as high as $23 \%$.

For 6 of 8 years, the effect of vaccination differed for individuals at high risk for influenza versus individuals at low risk for influenza, and age modified the effect of the vaccine in 4 of the 10 years. When data were pooled over all years, influenza vaccination appeared to be effective only in individuals $\leqslant 84$ of age, and it provided better protection to low-risk individuals (figure 2). A 21\% (RR, 0.79) effectiveness of vaccine against death due to respiratory disease was seen among low-risk patients aged 65-84 years, after adjustment for age and other confounders over 10 years (table 4). For high-risk individuals, the RR of death due to respiratory disease was 0.71 (95\% CI, 0.66-0.78) in the influenza season, but there also was an apparent effect in the periinfluenza season (RR, 0.8; 95\% CI, 0.74-0.87).

Attributable rate differences were calculated for individuals 6584 years of age in both risk categories, after standardization for age, sex, and repeat prescriptions. For low-risk individuals, the attributable rate reduction was 3.12 (95\% CI, 2.43-3.81) per 100,000 person-weeks in the influenza season, compared with 0.58 ( $95 \%$ CI, -0.42 to -1.21 ) in the peri-influenza season; for high-risk individuals who were 65-84 years of age, the attrib-

Table 1. Crude and adjusted rate ratios (RRs) of the effect of influenza vaccination against admissions to the hospital for acute respiratory disease from 1990-1991 to 1998-1999, by season.

\begin{tabular}{lcccc}
\hline & $\begin{array}{c}\text { Weeks of influenza } \\
\text { activity }^{\mathrm{a}} \text { (week of } \\
\text { peak activity) }\end{array}$ & Influenza season $^{\mathrm{b}}$ & Peri-influenza season $^{\mathrm{c}}$ & Summer season $^{\mathrm{d}}$ \\
\hline RR & & 1.18 & 1.40 & 1.48 \\
\hline $\begin{array}{l}\text { Pooled crude } \\
\text { Pooled adjusted }\end{array}$ & & $0.79(0.74-0.83)$ & $0.94(0.88-0.99)$ & $0.98(0.92-1.04)$ \\
Adjusted, by year & & & & \\
$1990-1991$ & $51-10(5)$ & $0.84(0.66-1.06)$ & $0.78(0.58-1.06)$ & $1.18(0.24-1.51)$ \\
$1991-1992$ & $51-09(2)$ & $0.70(0.59-0.82)$ & $1.15(0.95-1.38)$ & $1.06(0.88-1.27)$ \\
$1992-1993^{f}$ & $08-16(11)$ & $0.93(0.76-1.12)$ & $0.97(0.82-1.13)$ & $0.91(0.77-1.07)$ \\
$1993-1994$ & $42-51(45)$ & $0.76(0.62-0.94)$ & $0.95(0.83-1.08)$ & $0.95(0.81-1.12)$ \\
$1994-1995^{f}$ & $01-14(6)$ & $0.95(0.82-1.09)$ & $0.88(0.73-1.06)$ & $0.99(0.84-1.16)$ \\
$1995-1996$ & $45-03(48)$ & $0.69(0.59-0.80)$ & $0.98(0.84-1.15)$ & $1.05(0.88-1.24)$ \\
$1996-1997$ & $49-09(1)$ & $0.66(0.58-0.75)$ & $1.11(0.91-1.36)$ & $0.86(0.73-1.01)$ \\
$1997-1998^{f}$ & $6-14(7)$ & $0.97(0.79-1.17)$ & $0.95(0.81-1.1)$ & $1.14(0.95-1.37)$ \\
$1998-1999$ & $51-6(2)$ & $0.82(0.68-0.99)$ & $0.59(0.46-0.75)$ & $0.82(0.68-0.99)$ \\
\hline
\end{tabular}

NOTE. Data are RR or RR (95\% confidence interval).

a Week 1 started on the first Monday of each calendar year.

${ }^{b}$ Defined using general practitioner consultation rates for influenza-like illness that has been validated against the presence of circulating influenza virus in the same population [19]. In this surveillance system, the onset and the end of the influenza season occurred when consultation rates for influenza-like illness increased to $\geqslant 50$ consultations/100,000 person-weeks and decreased to $<50$ consultations/100,000 person-weeks, respectively [19].

${ }^{c}$ Defined as the weeks during the winter period that were outside the influenza season from 15 November each year to the end of April.

d Defined as the period from 1 May to the end of August each year.

e Pooled over 9 years.

f Years during which influenza activity was considered to be mild. 
Table 2. Rates of admission to the hospital for acute respiratory disease, per 1000 person-years, for vaccinated and unvaccinated cohorts, by age and risk group, as well as adjusted rate ratios (RRs) pooled over 9 years.

\begin{tabular}{|c|c|c|c|c|c|c|}
\hline \multirow[b]{2}{*}{ Finding } & \multicolumn{2}{|c|}{ Influenza season $^{a}$} & \multicolumn{2}{|c|}{ Peri-influenza season $^{\mathrm{b}}$} & \multicolumn{2}{|c|}{ Summer season ${ }^{c}$} \\
\hline & $\begin{array}{l}\text { Vaccinated } \\
\text { cohort }\end{array}$ & $\begin{array}{l}\text { Unvaccinated } \\
\text { cohort }\end{array}$ & $\begin{array}{l}\text { Vaccinated } \\
\text { cohort }\end{array}$ & $\begin{array}{l}\text { Unvaccinated } \\
\text { cohort }\end{array}$ & $\begin{array}{l}\text { Vaccinated } \\
\text { cohort }\end{array}$ & $\begin{array}{c}\text { Unvaccinated } \\
\text { cohort }\end{array}$ \\
\hline Person-years, no. & 145,706 & 274,042 & 187,625 & 349,785 & 245,338 & 447,077 \\
\hline $\begin{array}{l}\text { Among individuals at low risk for influenza, } \\
\text { by age group }\end{array}$ & $1993(13.68)$ & $3177(11.60)$ & $1955(10.40)$ & $2598(7.44)$ & $2009(8.16)$ & $2481(5.56)$ \\
\hline $65-69$ years & $53(2.81)$ & $191(2.76)$ & $52(2.13)$ & $146(1.66)$ & $72(2.18)$ & $156(1.35)$ \\
\hline 70-74 years & $84(3.90)$ & $217(4.16)$ & $87(3.12)$ & $192(2.81)$ & $88(2.39)$ & $207(2.34)$ \\
\hline $75-79$ years & $124(7.12)$ & $262(7.44)$ & $115(5.10)$ & $179(4.00)$ & $143(4.84)$ & $211(3.64)$ \\
\hline $80-84$ years & $131(11.18)$ & $320(13.78)$ & $132(8.79)$ & $233(7.90)$ & $136(6.92)$ & $224(5.98)$ \\
\hline$\geqslant 85$ years & $175(21.37)$ & $402(23.40)$ & $161(15.39)$ & $327(14.98)$ & $160(12.17)$ & $230(8.53)$ \\
\hline $\begin{array}{l}\text { Adjusted RR }(95 \% \mathrm{Cl}) \\
\text { Among individuals at high risk for influenza, } \\
\text { by age group }\end{array}$ & 0.7810 & 71-0.86) & $0.97(0$ & 37-1.07) & \multicolumn{2}{|c|}{$1.09(0.98-1.20)$} \\
\hline $65-69$ years & $170(11.08)$ & $214(11.18)$ & $196(9.93)$ & $193(7.90)$ & $195(7.49)$ & $195(6.19)$ \\
\hline 70-74 years & $281(15.91)$ & $280(15.03)$ & $249(10.97)$ & $262(10.97)$ & $278(9.31)$ & $241(7.96)$ \\
\hline $75-79$ years & $302(19.40)$ & $375(24.44)$ & $316(15.81)$ & $312(15.91)$ & $302(11.60)$ & $325(13.21)$ \\
\hline $80-84$ years & $316(28.24)$ & $436(35.93)$ & $325(22.67)$ & $359(23.30)$ & $325(17.68)$ & $320(16.85)$ \\
\hline$\geqslant 85$ years & $357(43.99)$ & $480(48.26)$ & $322(31.20)$ & $395(31.25)$ & $310(24.23)$ & $372(24.75)$ \\
\hline Adjusted RR (95\% Cl) & \multicolumn{2}{|c|}{$0.79(0.74-0.85)$} & \multicolumn{2}{|c|}{$0.92(0.85-0.99)$} & \multicolumn{2}{|c|}{$0.92(0.86-0.99)$} \\
\hline
\end{tabular}

a Defined using general practitioner consultation rates for influenza-like illness that has been validated against the presence of circulating influenza virus in the same population [19]. In this surveillance system, the onset and the end of the influenza season occurred when consultation rates for influenza-like illness increased to $\geqslant 50$ consultations/100,000 person-weeks and decreased to <50 consultations/100,000 person-weeks, respectively [19].

b Defined as the weeks during the winter period that were outside the influenza season from 15 November each year to the end of April.

c Defined as the period from 1 May to the end of August each year.

d Obtained by multiplying rates/1000 person-weeks by 52 to calculate annualized rates/1000 person-years.

utable rate reduction was 11.68 (95\% CI, 10.46-12.9) in the influenza season, 5.54 (95\% CI, 4.47-6.6) in the peri-influenza season, and 1.95 (95\% CI, 1.00-2.9) in the summer season.

\section{DISCUSSION}

We have shown that, during a number of epidemic and nonepidemic years, rates of hospitalization for acute respiratory disease were, on average, reduced to $21 \%$ (95\% CI, 17\%-26\%) when influenza vaccination was given to individuals $>64$ years of age after controlling for age and severity of underlying illness. For respiratory disease-associated death, the summary estimate was $12 \%$ (95\% CI, $8 \%-16 \%)$. We estimated vaccine effectiveness during the influenza season to increase the specificity of attribution of death or hospitalization to influenza. In general, no reduction in risk of disease was seen among influenza vaccine recipients in the peri-influenza season, when influenza was not assumed to be circulating. This finding confirmed that the overall effect of vaccination on acute respiratory disease, in terms of the risk of hospitalization or death, is not the result of underlying differences in vaccinated persons and unvaccinated persons in this population. For respiratory disease-associated death, however, there was a suggestion of some residual confounding in high-risk individuals, because a lower rate of respiratory disease-associated death was seen among vaccine recipients, compared with individuals who were not vaccinated, in the peri-influenza and summer seasons.

We used peri-influenza seasons as the baseline comparison, to assess the presence of unmeasured confounding. It is possible, however, that some of the deaths or hospitalizations that were associated with influenza occurred after the influenza season. A lag between the influenza season and outcomes was not assumed, because visual inspection of the numbers of deaths per week and the rates of admission to the hospital for acute respiratory disease indicated a peak occurring in association with the height of the epidemic period, by use of vital statistics in the United Kingdom [21] and data on admissions for acute respiratory disease in London, respectively [22].

Influenza vaccine is assumed to be less effective in individuals who are infirm, because the immune response to vaccine is not as strong in such individuals; however, this is not well established [23]. If the peri-influenza season effect is taken as the baseline with which to compare the relative effect of influenza vaccination, the present study demonstrated a greater relative protective effect against death due to respiratory disease among individuals at low risk for influenza, compared with individuals at high risk for influenza (for low-risk individuals, the RR was 0.79 , compared with an RR of 0.98 in the peri-influenza season; for high-risk individuals, the RR was 0.71 , compared with an $\mathrm{RR}$ of 0.8 in the peri-influenza season). The results also support 


\section{A}

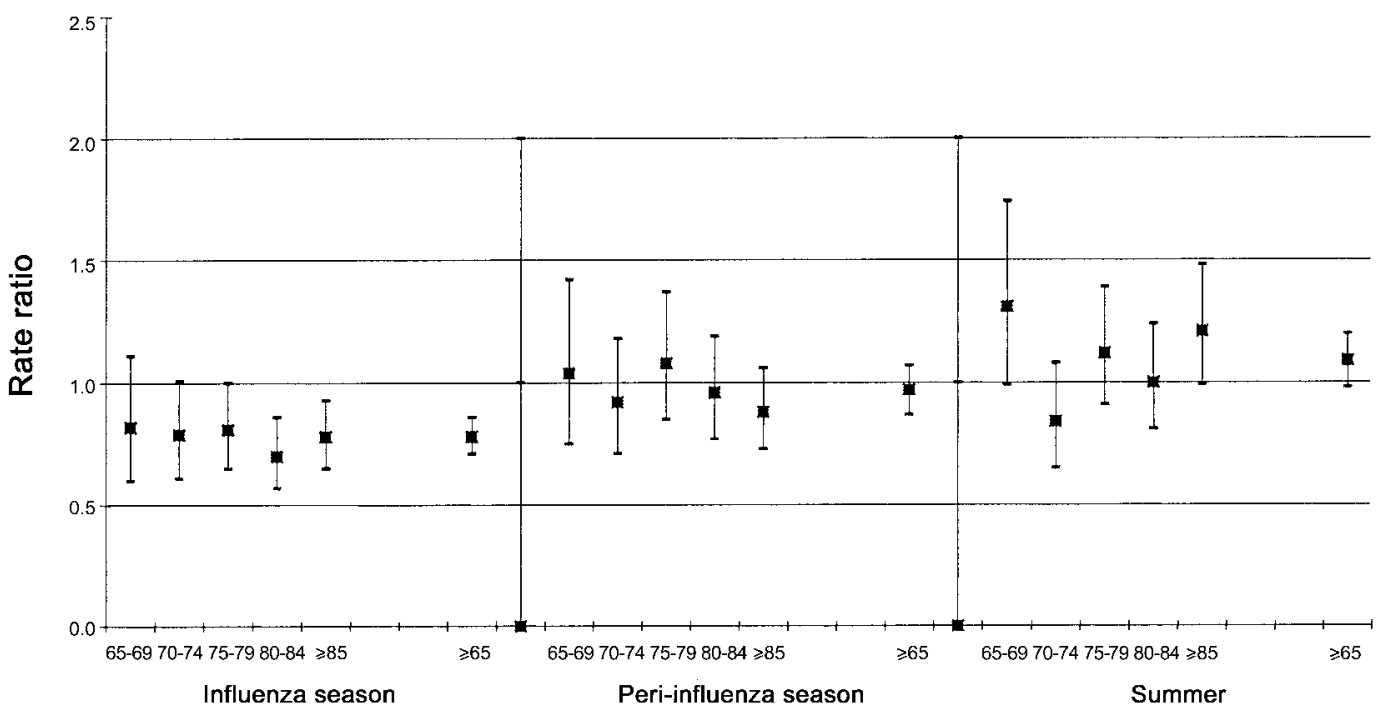

Age and season

B



Figure 1. Rate ratios (point estimates and 95\% confidence intervals) for hospitalizations for acute respiratory disease, adjusted for repeat prescription status, for individuals vaccinated against influenza, compared with individuals not vaccinated against influenza, stratified by age group and season pooled over 9 years. $A$, Individuals at low risk for influenza; $B$, individuals at high risk for influenza.

an age-based policy for vaccination, because they confirm, for older people, the importance of influenza vaccination in reducing severe complications associated with influenza, regardless of underlying medical disorders.

Data for patients for whom information on smoking is available in general-practice records are known to be biased toward individuals with medical conditions [24] and individuals who are health-care seekers, and, so, they could not be used to control directly for confounding. Comparison of the effective- ness of influenza vaccine in the influenza season with the effectiveness of influenza vaccine in the peri-influenza season instead allowed control of unmeasured confounders, including both smoking and socioeconomic status, which would otherwise act to overestimate vaccine effectiveness. It is well known that individuals who receive influenza vaccination are less likely to smoke than are individuals who do not receive vaccination and that smokers are more likely to get influenza [25]. As with most preventive interventions, vaccination is less likely among 
Table 3. Crude and adjusted rate ratios (RRs) of the effect of influenza vaccination against death due to respiratory disease from 1989-1990 to 1998-1999, by season.

\begin{tabular}{|c|c|c|c|c|}
\hline $\mathrm{RR}$ & $\begin{array}{l}\text { Weeks of influenza } \\
\text { activity }^{\text {a }} \text { (week of } \\
\text { peak activity) }\end{array}$ & Influenza season ${ }^{b}$ & Peri-influenza season $^{c}$ & Summer season $^{d}$ \\
\hline Pooled crude & & 1.32 & 1.49 & 1.52 \\
\hline Pooled adjusted & & $0.88(0.84-0.92)$ & $1.00(0.95-1.05)$ & $1.01(0.96-1.06)$ \\
\hline \multicolumn{5}{|l|}{ Adjusted, by year } \\
\hline 1989-1990 & 46-03 (49) & $0.8(0.51-1.25)$ & $1.33(0.85-2.07)$ & $1.02(0.67-1.55)$ \\
\hline 1990-1991 & $51-10(5)$ & $0.81(0.66-0.99)$ & $0.96(0.74-1.26)$ & $1.09(0.88-1.35)$ \\
\hline 1991-1992 & $51-09(2)$ & $0.96(0.83-1.11)$ & $1.16(0.98-1.36)$ & $0.97(0.82-1.13)$ \\
\hline 1992-1993 & 08-16 (11) & $0.87(0.74-1.03)$ & $1.17(1.02-1.33)$ & $1.14(0.99-1.31)$ \\
\hline 1993-1994 & 42-51 (45) & $0.84(0.69-1.03)$ & $0.99(0.88-1.11)$ & $1.15(1.0-1.32)$ \\
\hline 1994-1995 & 01-14 (6) & $1.04(0.92-1.18)$ & $1.04(0.88-1.23)$ & $1.01(0.88-1.16)$ \\
\hline 1995-1996 & 45-03 (48) & $0.77(0.68-0.89)$ & $1.01(0.88-1.15)$ & $1.05(0.9-1.22)$ \\
\hline 1996-1997 & 49-09 (1) & $0.87(0.77-0.97)$ & $0.89(0.74-1.07)$ & $0.88(0.76-1.03)$ \\
\hline 1997-1998 & 6-14 (7) & $1.02(0.85-1.23)$ & $0.92(0.79-1.06)$ & $1.00(0.86-1.16)$ \\
\hline 1998-1999 & $51-6(2)$ & $0.79(0.65-0.96)$ & $0.82(0.66-1.03)$ & $1.00(0.80-1.25)$ \\
\hline
\end{tabular}

NOTE. Data are RR or RR (95\% confidence interval).

a Week 1 started on the first Monday of each calendar year.

b Defined using GP consultation rates for influenza-like illness that has been validated against the presence of circulating influenza virus in the same population [19]. In this surveillance system, the onset and the end of the influenza season occurred when consultation rates for influenza-like illness increased to $\geqslant 50$ consultations/100,000 person-weeks and decreased to $<50$ consultations/100,000 person-weeks, respectively [19].

${ }^{c}$ Defined as the weeks during the winter period that were outside the influenza season from 15 November each year to the end of April.

d Defined as the period from 1 May to the end of August each year.

e Pooled over 10 years.

individuals in lower socioeconomic groups. Such individuals are also likely to have higher morbidity and mortality rates.

Other research groups, which have controlled for unmeasured confounders by comparing effects in influenza seasons with effects in noninfluenza seasons, have shown a similar effectiveness of vaccine against hospitalizations for pneumonia and influenza. For example, in a US health maintenance organization (HMO) population, the effectiveness of vaccine against hospitalizations for pneumonia and influenza was 31\% (95\% CI, 4\%-51\%) in the influenza season of 1990-1991, compared with a vaccine effectiveness of $2 \%$ (95\% CI, $-39 \%$ to $31 \%$ ) in the peri-influenza winter season of the same year [26]. A recent cohort analysis of 3 other US HMO populations also showed that the effectiveness of vaccine against admissions to the hospital for pneumonia and influenza in 1998-1999 was $32 \%$ (95\% CI, 22\%-40\%). No significant reduction in the risk of hospitalization in general was noted in the summer [5]. Several other observational studies and a meta-analysis of observational studies performed in 1995 [27] have also noted a similar effect; however, in these studies, there were no comparisons with a noninfluenza season to exclude potential bias from unmeasured differences between vaccine recipients and individuals who did not receive vaccine.

A possible limitation is the specificity of respiratory diseaseassociated deaths as a marker of influenza-related death. Causeof-death data in the GPRD are, however, likely to be more specific than data on death certificates. GPs use their own judgment regarding the clinical information recorded. The imme- diate cause of the event is more relevant than the underlying cause, because the vaccine works by preventing acute respiratory illness and associated complications.

To allow generalizability, we calculated the rates of death or hospitalization that would be prevented by a program of influenza vaccination with full coverage. For example, a rough indication of the attributable rate reduction that is attributable just to influenza vaccination for hospitalizations can be calculated. The attributable rate reduction in the influenza season minus the attributable rate reduction in the peri-influenza season, multiplied by an average annual influenza period of 9.6 weeks in the 1990s, provides an estimate of 39.8 (i.e., [5.441.29] $\times 9.6)$ prevented hospitalizations $/ 100,000$ persons $>64$ years of age/year, with a program of full vaccination coverage. In England and Wales, the effect of full influenza vaccination would be equivalent to 3300 prevented hospitalizations/year. The total expected number of excess hospitalizations associated with respiratory disease attributed to influenza, in England and Wales, is 9000 excess hospitalizations/year (or 125 excess hospitalizations/100,000 persons $>64$ years of age/year) [21]. This yearly average is based on national data for 1990-1991 to 19961997, when vaccine uptake ranged from $20 \%$ to $47 \%$ among individuals $>64$ years of age [28].

In the present study, rates of hospitalization due to acute respiratory disease and all causes appear to be lower, by $\sim 20 \%$, than national rates for this age group. Although this would not affect the relative effectiveness of the vaccine, it would underestimate the absolute rate reduction, as would the fixed number 


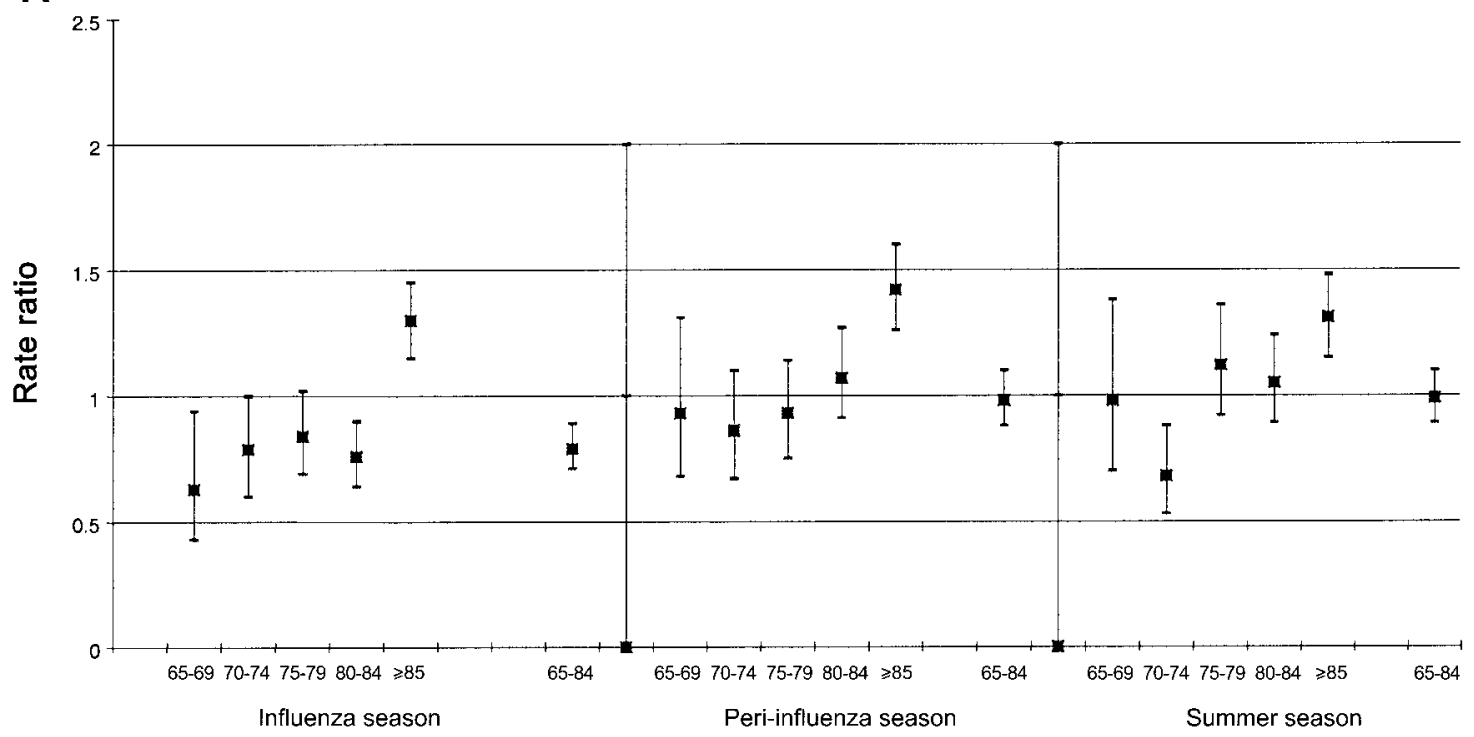

B

Age and season

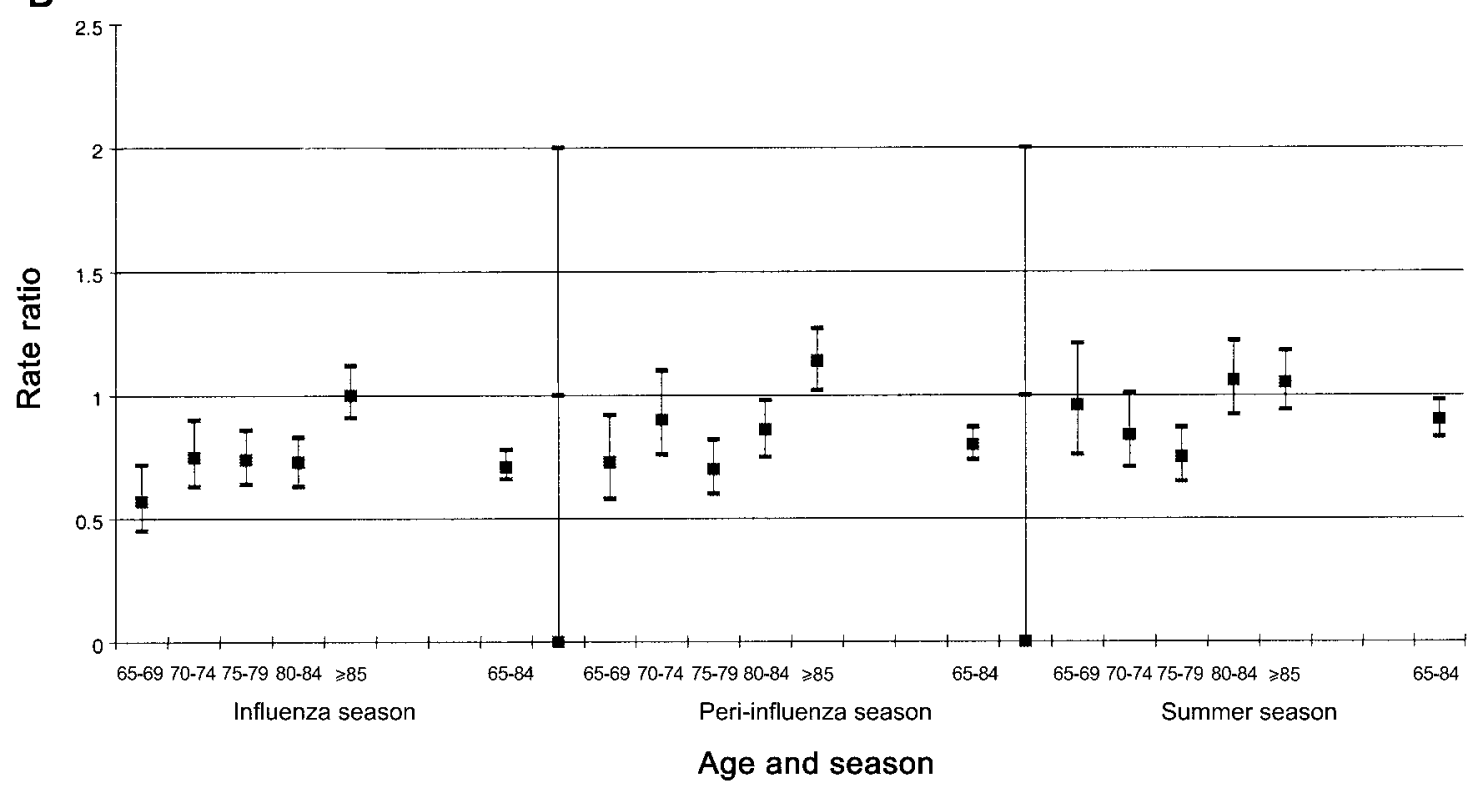

Figure 2. Rate ratios (point estimates and 95\% confidence intervals) for death due to respiratory disease, adjusted for repeat prescription status, for individuals vaccinated against influenza, compared with individuals not vaccinated against influenza, stratified by age group and season pooled over 10 years. $A$, Individuals at low risk for influenza; $B$, individuals at high risk for influenza.

of beds available. Other studies find hospitalization data in the GPRD to be quite complete. For instance, $87 \%$ of diagnoses from all subsequent hospital referrals for new nonsteroidal antiinflammatory drug users and $97.5 \%$ of all subsequent hospitalization discharge letters for antidiabetic-drug users were noted in the GPRD $[12,13]$. For $90 \%$ of the diagnoses, the same 3-digit ICD-9 code recorded at admission to the hospital was present in the hospital discharge letter. It is possible that some hospitalizations resulting in deaths of individuals in this age group may not be recorded. Sixty percent of death certificates for individuals $>64$ years of age are completed by hospital doctors [29]. However, in this data set, only $39 \%$ of individuals had a hospitalization during the month before death; $47 \%$ of individuals had a hospitalization during the 6 months before death (C. Hodgson, unpublished data).

In 1998-1999, vaccination seemed to have little effect against admissions to the hospital for respiratory disease; the reasons for this finding are unclear. The summary estimate was, how- 
Table 4. Rates of death due to respiratory disease/1000 person-years in vaccinated and unvaccinated cohorts, by age and risk group, and adjusted rate ratios (RRs) pooled over 10 years.

\begin{tabular}{|c|c|c|c|c|c|c|}
\hline \multirow[b]{2}{*}{ Finding } & \multicolumn{2}{|c|}{ Influenza season ${ }^{a}$} & \multicolumn{2}{|c|}{ Peri-influenza season ${ }^{b}$} & \multicolumn{2}{|c|}{ Summer season ${ }^{c}$} \\
\hline & $\begin{array}{l}\text { Vaccinated } \\
\text { cohort }\end{array}$ & $\begin{array}{l}\text { Unvaccinated } \\
\text { cohort }\end{array}$ & $\begin{array}{l}\text { Vaccinated } \\
\text { cohort }\end{array}$ & $\begin{array}{l}\text { Unvaccinated } \\
\text { cohort }\end{array}$ & $\begin{array}{l}\text { Vaccinated } \\
\text { cohort }\end{array}$ & $\begin{array}{l}\text { Unvaccinated } \\
\text { cohort }\end{array}$ \\
\hline Person-years, no. & 147,294 & 279,374 & 190,226 & 357,744 & 248,427 & 456,514 \\
\hline \multicolumn{7}{|l|}{ Admissions to the hospital, no. (annualized rates ${ }^{d}$ ) } \\
\hline Total & $2585(17.52)$ & $3720(13.31)$ & $2650(13.94)$ & $3336(9.31)$ & $2699(10.87)$ & $3266(7.18)$ \\
\hline \multicolumn{7}{|l|}{$\begin{array}{l}\text { Among individuals at low risk for influenza, } \\
\text { by age group }\end{array}$} \\
\hline 65-69 years & $31(1.61)$ & $136(1.92)$ & $44(1.77)$ & $130(1.40)$ & $47(1.40)$ & $129(1.09)$ \\
\hline 70-74 years & $74(3.38)$ & $186(3.43)$ & $91(3.22)$ & $208(2.96)$ & $83(2.24)$ & $234(2.60)$ \\
\hline 75-79 years & $145(8.22)$ & $285(7.90)$ & $137(5.98)$ & $241(5.20)$ & $174(5.72)$ & $247(4.16)$ \\
\hline 80-84 years & $191(16.12)$ & $423(17.89)$ & $228(14.98)$ & $354(11.70)$ & $239(12.01)$ & $368(9.62)$ \\
\hline$\geqslant 85$ years & 491 (59.33) & $661(37.28)$ & $477(45.08)$ & $583(26.16)$ & $446(33.49)$ & $578(21.11)$ \\
\hline Adjusted $R^{e}$ & 0.7910 & $71-0.89)$ & 0.9810 & $88-1.10)$ & 0.9910 & $89-1.10)$ \\
\hline \multicolumn{7}{|l|}{$\begin{array}{l}\text { Among individuals at high risk for influenza, } \\
\text { by age group }\end{array}$} \\
\hline $65-69$ years & $107(7.38)$ & $190(9.72)$ & $124(6.4)$ & $171(6.97)$ & $141(5.41)$ & $142(4.63)$ \\
\hline 70-74 years & $226(12.95)$ & $271(14.51)$ & $239(10.45)$ & $234(9.83)$ & $248(8.53)$ & $254(8.37)$ \\
\hline 75-79 years & $318(20.64)$ & $371(24.23)$ & $294(14.77)$ & $358(18.36)$ & $335(13.1)$ & $368(14.87)$ \\
\hline $80-84$ years & $358(31.77)$ & $480(39.88)$ & $397(28.03)$ & $446(28.7)$ & $429(23.04)$ & $376(19.40)$ \\
\hline$\geqslant 85$ years & $644(78.31)$ & 717 (71.40) & 619 (60.37) & $611(48.41)$ & 557 (43.06) & 570 (37.60) \\
\hline Adjusted RR $\mathrm{R}^{\mathrm{e}}$ & \multicolumn{2}{|c|}{$0.71(0.66-0.78)$} & \multicolumn{2}{|c|}{$0.80(0.74-0.87)$} & \multicolumn{2}{|c|}{$0.90(0.83-0.98)$} \\
\hline \multicolumn{7}{|c|}{$\begin{array}{l}\text { a Defined using general practitioner consultation rates for influenza-like illness that has been validated against the presence of circulating influenza virus in the same } \\
\text { opulation [19]. In this surveillance system, the onset and the end of the influenza season occurred when consultation rates for influenza-like illness increased to } \geqslant 50 \\
\text { nsultations } / 100,000 \text { person-weeks and decreased to }<50 \text { consultations/100,000 person-weeks, respectively [19]. } \\
\text { b Defined as the weeks during the winter period that were outside the influenza season from } 15 \text { November each year to the end of April. } \\
\text { c Defined as the period from } 1 \text { May to the end of August each year. } \\
\text { d Rates per } 1000 \text { person-years. } \\
\text { e Calculated excluding data for individuals } \geqslant 85 \text { years of age. }\end{array}$} \\
\hline
\end{tabular}

ever, insensitive to the removal of data for 1998-1999. Pooled over 8 years, the summary estimate was $23 \%$ (95\% CI, $18 \%-$ $27 \%)$ in the influenza season and $3 \%(95 \% \mathrm{CI},-3 \%$ to $11 \%)$ in the peri-influenza season.

The present study indicates an effect of influenza vaccine in preventing deaths due to respiratory disease. This finding is similar to the findings of 1 or 2 other studies. One of these studies, a US HMO cohort study with a total of only 100 deaths, found that the effectiveness of vaccine against death, following hospitalization for pneumonia and influenza, was 33\% (95\% CI, $-7 \%$ to $58 \%$ ) [30].

The RR for death due to all causes, pooled over 10 years, was $0.79(95 \% \mathrm{CI}, 0.77-0.81)$ in the influenza season. It was similar to that seen in the peri-influenza season $(0.81 ; 95 \% \mathrm{CI}$, $0.79-0.83)$. The estimate for the summer season was 0.88 (95\% CI, 0.86-0.90). These findings are consistent with vaccine nonrecipients being more vulnerable to winter infections other than influenza. The RRs in the influenza and peri-influenza seasons were similar for all years except 1995-1996, when protection offered by the influenza vaccine against deaths due to respiratory disease was high (data not presented).

Other cohort studies have not compared the effect of the vaccine on death due to all causes in the influenza season with that noted for the peri-influenza season [31, 32]. Confounding by a healthy vaccinee effect is possible because, for instance, prevalence of smoking was lower and education level was higher for vaccine recipients, compared with individuals who did not receive vaccine, in one such cohort study [32]. We compared the effect of influenza vaccine in the influenza season with the effect of influenza vaccine in the peri-influenza season, to assess the possibility of bias resulting in overestimation of vaccine effectiveness. We also attempted to control for confounding by indication, whereby individuals at higher risk are more likely to be vaccinated, by using the number of repeat prescriptions as a marker of the severity of underlying risk conditions. However, it may be that there was still some unmeasured confounding that underestimated vaccine effectiveness against overall mortality. This residual confounding is likely to be the explanation for the higher rate of deaths due to respiratory disease seen among individuals $>84$ years of age (e.g., because many such individuals are in nursing homes, where vaccination coverage is much higher), compared with individuals at younger ages (or between 65 and 84 years of age).

In the present study, the specificity of influenza-associated death or hospitalization has been increased by limiting events to time periods when influenza has been shown to be circulating. Cold weather is thought to be a direct cause of excess morbidity and deaths occurring in the winter. Respiratory syn- 
cytial virus also often occurs at the same time that influenza occurs each year [33]. Analyses of the role of influenza vaccination controlling for climate and for climate-related factors would give an even more specific case definition for influenzarelated outcomes.

Using vital statistics for the 1990s, Fleming [21] observed that, during influenza epidemics, premature deaths of frailer individuals who would have died later during the year anyway were not occurring, to any extent, apart from during the epidemic year 1989-1990. People are therefore dying in the winter periods, and these deaths are preventable by use of influenza vaccine. It is not possible to predict epidemics, and vaccine effectiveness will vary depending on the "match" between circulating strains and the current vaccine used that year. During the study period here, a good match in general was achieved. Our estimate of the effect of influenza vaccine in a UK population, averaged over a number of epidemic and nonepidemic years, strengthens the case for influenza vaccination for all older people with or without underlying medical disorders.

\section{Acknowledgments}

We thank the staff at the Respiratory Diseases Department, UK Health Protection Agency (London) for access to and discussions about the initial list of Oxford Medical Information System codes used to assign patients to the high-risk category and for information on the influenza seasons. We also thank Simon Cousens for advice on the calculation of differences in the standardized rates.

\section{References}

1. Ashley J, Smith T, Dunnell K. Deaths in Great Britain associated with the influenza epidemic of 1989/90. Popul Trends 1991;65:16-20.

2. Glezen WP, Decker M, Perrotta DM. Survey of underlying conditions of persons hospitalized with acute respiratory disease during influenza epidemics in Houston, 1978-1981. Am Rev Respir Dis 1987; 136:550-5.

3. Barker WH, Mullooly JP. Pneumonia and influenza deaths during epidemics: implications for prevention. Arch Intern Med 1982; 142:85-9.

4. Curwen M, Dunnell K, Ashley J. Hidden influenza deaths. BMJ 1990; 300:896.

5. Nichol KL, Nordin J, Mullooy J, Lask R, Fillbrandt K, Iwane M. Influenza vaccination and reduction in hospitalizations for cardiac disease and stroke among the elderly. N Engl J Med 2003;348:1322-32.

6. Fleming DM, Watson JM, Nicholas S, Smith GE, Swan AV. Study of the effectiveness of influenza vaccination in the elderly in the epidemic of 1989-90 using a general practice database. Epidemiol Infect 1995; 115:581-9.

7. Christensen B, Lundbergh P, Hedlund J, Ortqvist A. Effects of a largescale intervention with influenza and 23 -valent pneumococcal vaccines in adults aged 65 years and older: a prospective study. Lancet 2001; 357:1008-11.

8. Govaert TM, Thijs CT, Masurel N, Sprenger N, Dinant GJ, Knottnerus JA. The efficacy of influenza vaccination in elderly individuals: a randomized double-blind placebo-controlled trial. JAMA 1994;272:1661-5.

9. Dedman DJ, Zambon MC, Van Buynder P, Fleming DM, Watson JM, Joseph CA. Influenza surveillance in England and Wales: October 1997 to June 1998. Commun Dis Public Health 1998; 1:244-51.

10. Whiting P, Joseph CA, Zambon M, Nunn M, Fleming DM, Watson
JM. Influenza activity in England and Wales: October 1998 to June 1999. Communicable Disease and Public Health 1999;2:273-9.

11. Lawson DH, Sherman V, Hollowell J. The general practice research database: scientific and ethical advisory group. Q J Med 1998; 91 : $445-52$.

12. Jick H, Jick SS, Derby LE. Validation of information recorded on general practitioner based computerised data resource in the United Kingdom. BMJ 1991;302:766-8.

13. Van Staa JT, Abenhaim L. The quality of information recorded on a UK database of primary care records: a study of hospitalisations due to hypoglycaemia and other conditions. Pharmacoepidemiology and Drug Safety 1994; 3:15-21.

14. Nazareth I, King M, Haines A, Rangel L, Myers S. Accuracy of diagnosis of psychosis on general practice computer system. BMJ 1993;307:32-4.

15. Office for National Statistics. Key health statistics from general practice 1996. Series MB6, no. 1. London: Office for National Statistics, 1998.

16. Office for National Statistics. Key health statistics from general practice 1998. Series MB6, no 2. London: Office for National Statistics, 2000.

17. Salisbury D, Begg NT, eds. 1996 Immunisation against infectious disease. London: Her Majesty's Stationery Office, 1996.

18. British national formulary. 44th ed. London: Pharmaceutical Press, 2002, ch. 2-6, 8, and 9.

19. Fleming DM, Zambon M, Bartelds AI, de Jong JC. The duration and magnitude of influenza epidemics: a study of surveillance data from sentinel general practices in England, Wales and the Netherlands. Eur J Epidemiol 1999; 15:467-73.

20. Stata Corporation. Intercooled Stata 7. Texas: Stata Press, 2001.

21. Fleming DM. The contribution of influenza to combined acute respiratory infections, hospital admissions and deaths in winter. Communicable Disease and Public Health 2000;3:32-8.

22. Better care of respiratory illness could reduce winter pressures. BMJ 2002; 324:66.

23. Iorio AM, Alatri A, Camilloni B, Neri M, Baglio G, Donatelli I. Antibody response to 1995-1996 influenza vaccine in institutionalized and non-institutionalized elderly women. Gerontology 1999; 45:31-8.

24. Mant J, Murphy M, Rose P, Vessey M. The accuracy of general practitioner records of smoking and alcohol use: comparison with patient questionnaires. J Public Health Med 2000; 22:198-201.

25. Nicholson KG, Kent J, Hammersley V. Influenza A among communitydwelling elderly persons in Leicestershire during winter 1993-4: cigarette smoking as a risk factor and the efficacy of influenza vaccination. Epidemiol Infect 1999; 123:103-8.

26. Ohmit SE, Monto AS. Influenza vaccine effectiveness in preventing hospitalization among the elderly during influenza type A and type B seasons. Int J Epidemiol 1995; 24:1240-8.

27. Gross PA, Hermogenes AW, Sacks HS, Lau J, Levandowski RA. The efficacy of influenza vaccine in elderly persons: a meta-analysis and review of the literature. Ann Intern Med 1995; 123:518-27.

28. Irish C, Alli M, Gilham C, Joseph C, Watson J. Influenza vaccine uptake and distribution in England and Wales, July 1989-June 1997. Health Trends 1998; 30:51-5.

29. Mortality statistics general: England and Wales, 1993-1995. DH1, no. 28, table 3. London: Office of National Statistics, 1997.

30. Mullooly JP, Bennett MD, Hornbrook MC, et al. Influenza vaccination programs for elderly persons: cost-effectiveness in a health maintenance organization. Ann Intern Med 1994; 121:947-52.

31. Voordouw BCG, van der Linden PD, Simonian S, et al. Influenza vaccination in community dwelling elderly: impact on mortality and influenza-associated morbidity. Arch Intern Med 2003; 163:1089-94.

32. Christensen B, Lundbergh P. Comparison between cohorts vaccinated and unvaccinated against influenza and pneumococcal infection. Epidemiol Infect 2002; 129:515-24.

33. Zambon MC, Stockton JD, Clewley JP, Fleming DM. Contribution of influenza and respiratory syncytial virus to community cases of influenza-like illness: an observational study. Lancet 2001;358:1410-6. 